\title{
Adaptive Noise cancellation using Binary LMS Algorithm
}

\author{
N.Haritha ${ }^{1}$, S.Venkata swamy ${ }^{2}$ \\ M.Tech Student Department of Electronics and Communications, Sarada Institute of Science Technology \\ and Management(SISTAM), Srikakulam, AP, India ${ }^{1}$
}

Associate professor Department of Electronics and Communications, Sarada Institute of Science Technology and Management(SISTAM), Srikakulam, AP, India $^{2}$

\begin{abstract}
In Speech processing Signal to Noise (SNR )Ratio plays an important role for Noise cancellation. here an attempt is made to evaluate SNR for given Noisy Speech signal using adaptive technique Binary LMS algorithm. In this proposal , Introduces the two control parameters of the Adaptive Algorithm rather than one control parameter as in case of LMS Algorithm. Also comparirison is made with respect to the

SNR for the given noisy speech.
\end{abstract}

Keywords: Adaptive Filters, Windows,LMS, and Binary LMS

\section{INTRODUCTION}

Digital filter plays an important role in digital signal activities[9].VF is raditionally described as a system of processing applications. Digital filters are widely used in many chaotic in the myocardium wandering, electrical digital signal processing applications, such as digital wavelets, ever changing in direction and number In signal filtering, noise filtering, signal frequency analysis, contrast, recent findings indicate that stable organized speech and audio compression, biomedical signal centres of rapid activity, called "mother rotors" [10]. processing and image enhancement etc.[1]. Traditionally, most digital filter applications have been limited to audio and high-end image processing. With advances in process technologies and digital signal processing methodologies, digital filters are now cost-effective in the IF range and in almost all video markets[2].Finite impulse response (FIR) digital filter impulse response is finite, so it can be used for Fast Fourier Transform (FFT) algorithm to achieve the filtered signal, which can greatly improve the efficiency of operation. In addition, FIR digital filter can be designed a linear phase digital filter which is convenient for image processing and data transmission applications[3]FIR Filter banks are used to perform short-term spectram Analysis in a variety of speech processing systems[4]. Many Window functions are widely used in digital signal processing for various applications in signal analysis and estimation, digital filter design and speech processing[5]. embedded devices.Heart rate frequency is very important health status information. The frequency measurement is used in many medical or sport applications like stress tests or life treating situation prediction.[6]. The accurate extraction of the AA signal from the ECG of AF is of great interest for subsequent analysis, since it has been documented to provide significant information on the properties of $\mathrm{AF}$ episodes[7]. reduction represents another important objective of ECG signal processing; in fact, the waveforms of interest are sometimes so heavily masked by noise that their presence can only be revealed once appropriate signal rocessing has first been applied.[8] In the last two decades, spectral analysis of the residual ECG signal (rECG, i.e. an ECG signal in which ventricular components were canceled through beat averaging techniques) has been employed to characterize atrial

\section{ADAPTIVE FILTERS\&LMS ALGORITHM} Adaptive filters provide performance excellence due to their inherent pole-zero structure as compared with adaptive finite impulse response (FIR) filters that have an all-zero form ,in active noise control Application[11].RLS Filters[12].Adaptive Filters are highly stable and effectively attenuate and often cancel destitutions[13].An Adaptive filters are successfully used in bio-medical processing systems like Denoising of ECG Waveforms[14] Adaptive filters play an important role in modern Digital signal processing products in area such as telephone echo cancellations ,noise cancellation, equalization of communications channels, biomedical signal enhancement, active noise control, and adaptive control systems[15] and many Authors are worked out on FIR Filters using different Transform techniques[16] to [20]

\section{DESIGN OF LMS BASED ADAPTIVE FILTER}

In our illustrative numerical example, the adaptive filter is set to be a 100-tap FT based FIR filter to simplify numerical algebra. The filter adjustable coefficient wn is adjusted based on the LMS algorithm .

$$
w_{n+1}=w_{n}+m * e(n) x(n)---(3)
$$

where wn is the coefficient used currently, while $w_{n+1}$ is the coefficient obtained from the LMS algorithm and will be used for the next coming input sample. The value of $m$ controls the speed of the coefficient change, $e(n)$ is an error value updated each time and $x(n)$ is noised signal coefficient. The output equations of LMS algorithm leads to 


$$
F(n)=G(n) * x(n)---(4)
$$$$
e(n)=d(n)-y(n)---(5)
$$$$
G_{n+1}=G+m * e(n) x(n)--(6)
$$

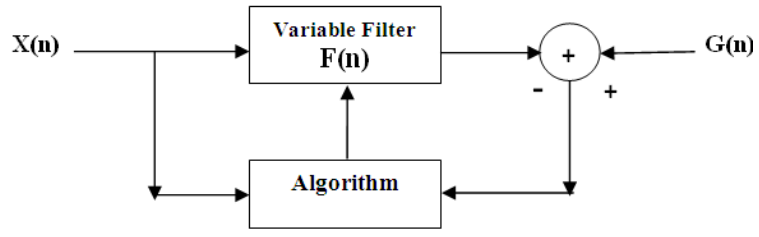

Fig1: Block diagram of Adaptive filter.

\subsection{Steps to Design Adaptive Filter}

1) The Low pass filter removes the corrupting low frequency noises in signal. The order of the filter is The order of the filter is 64 .

Steps to low pass filter:

The desired transfer function of filter is

$$
h_{d}(n)=\frac{\sin w_{c} n}{\pi n}
$$

By multiplying the desired transfer function with windows designed by FrFT, we can get transfer function of FIR band reject filter i.e

$$
h(n)=h_{d}(n) * w(n)
$$

where $w(n)$ is represents Transfer function of following windows

1) Rectangle window

2) Bartlett window
3) Hanning window
4) Hamming window
5) Kaiser window

2) Now $h(n)$ is compared with $x(n)$ which produces $e(n)$.

3) The error coefficients are fed back to LMS algorithm to update the coefficients of FrFt based LPF.

4) Steps 2 and 3 repeated up to error becomes negligible.

5) The updated coefficients of LMS Algorithm is the is the Response of desired Filter

\section{DESIGN OF BINARY LMS BASED ADAPTIVE FILTER}

It was Evaluated out modifications of the LMS algorithm . Getting to one of the drawbacks of LMS, that it has only one controllable parameter "mu", the selection of whose value will be the most critical from design point of view with Respect to. convergence. So, Here implementation of LMS in such a way that the step-size adapts to the error occurring in each iteration.

In Binary Step-size LMS algorithm, we have two step sizes calculated from 2 values, delta and deviation. When the error increases from the previous value of error, step size is (delta deviation). And when the error decreases from its previous value, step size is (delta-deviation). And finally implemented an adaptive Filter using the BS-LMS algorithm. And Design Considerations are same as above as Discussed in Section-3.

\section{RESULTS AND IMPLEMENTATIONS}

The results shows responses of the FrFT based Adaptive filter with LMs Algorithm and we applied a noised signal shown in Fig2 and compares the signal to noise ratio of Noised signal before and after the filtering for different Fractional Parameters of FrFT.

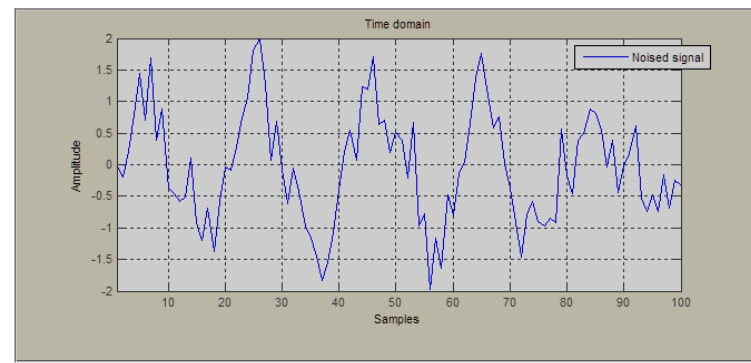

Fig2: Noised signal

When the Noised signal of fig-2 is filtered with Adaptive Filter with both LMS and Binary LMS algorithms the whole noise was removed, producing a near clean signal of fig:3 to fig:10 with different window combinations of Desired FrFt based Filter. and SNR values of noised and denoised signals are calculated for LMs and Binary LMS Algorithms for different windows are shown in Table-1

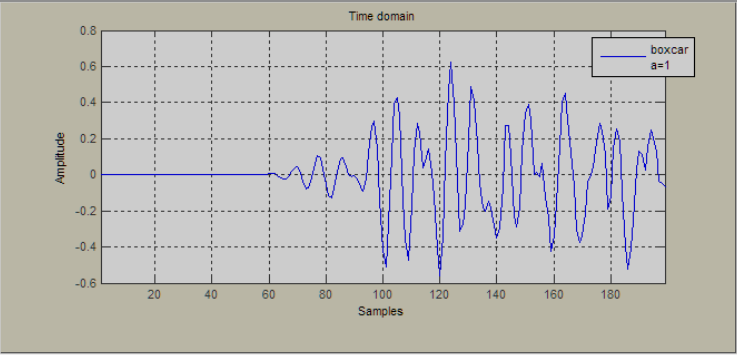

Fig3:Response of LMS based Adaptive filter with Rectangular window

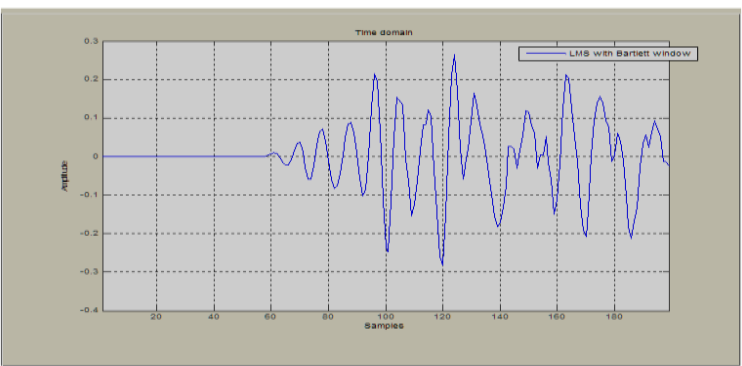

Fig4:Response of LMS based Adaptive filter with Bartlett window

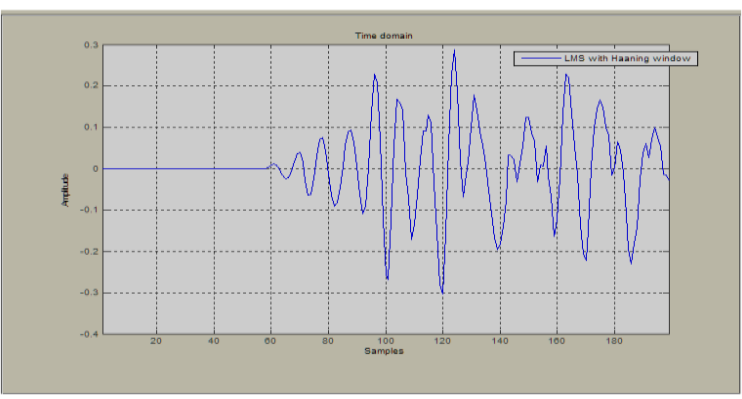

Fig5: Response of LMS based Adaptive filter with Hanning window 


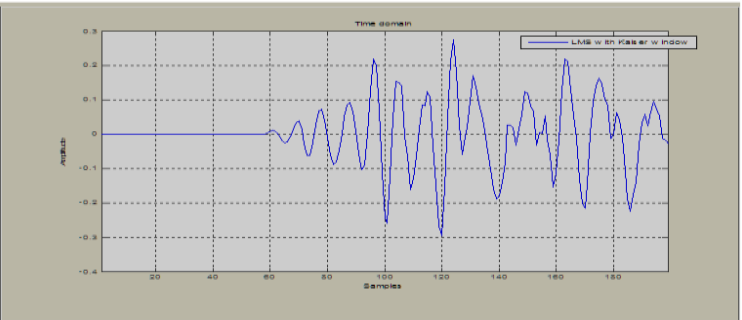

Fig6:Response of LMS based Adaptive filter withkaiserwindow

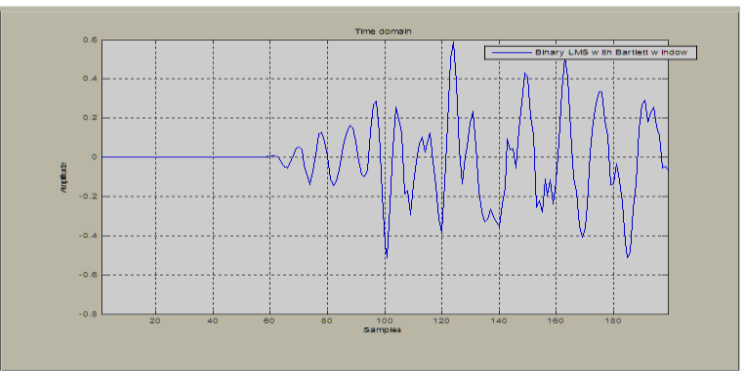

Fig7: Response of Binary LMS based Adaptive filter with Bartlett window

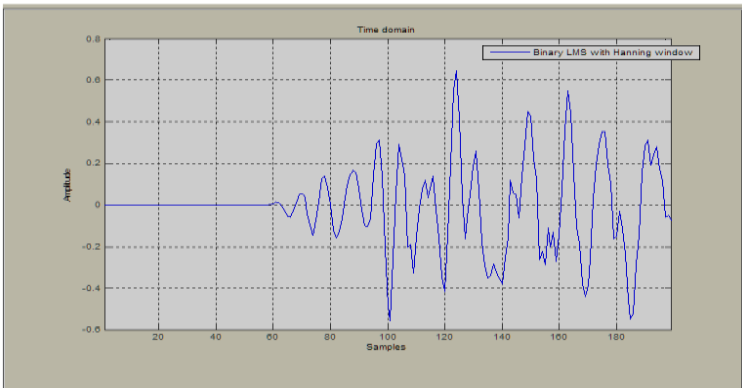

Fig8: Response of Binary LMS based Adaptive filter with Hanning window

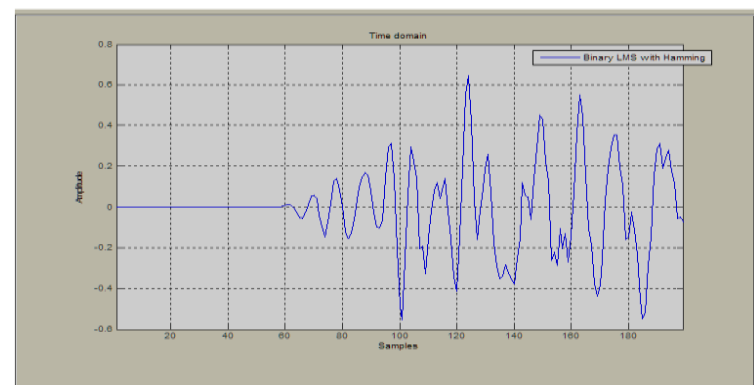

Fig9: Response of Binary LMS based Adaptive filter with Hamming window

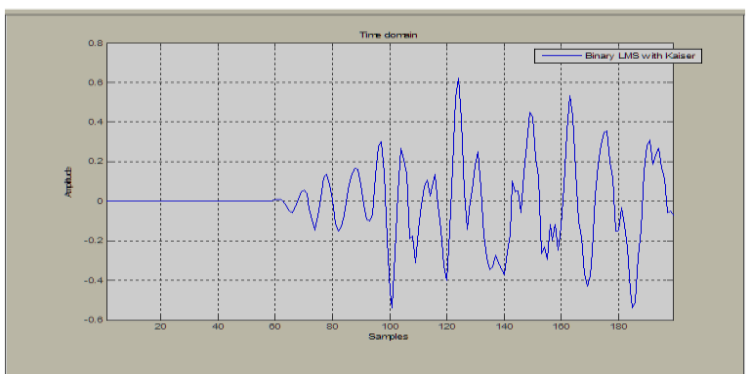

Fig10: Response of Binary LMS based Adaptive filter with Kaiser window
Table1:SNR calculation for LMS and Binary LMS for different windows

\begin{tabular}{|l|l|l|}
\hline Window & $\begin{array}{l}\text { SNR in DB for } \\
\text { LMS Algorithm }\end{array}$ & $\begin{array}{l}\text { SNR in dB for } \\
\text { Binary LMS } \\
\text { Algorithm }\end{array}$ \\
\hline Rectangle & 12.9576 & 28.2637 \\
\hline Bartlett & 11.4899 & 26.7395 \\
\hline Hanning & 12.9468 & 28.2315 \\
\hline Hamming & 12.9312 & 28.2169 \\
\hline kaiser & 12.4831 & 27.7149 \\
\hline
\end{tabular}

\section{CONCLUSION}

The Implementation of Adaptive-FIR Filter using LMS Algorithm and Binary LMS Algorithm with Different Digital windows was performed. and we also applied a sample test noised signal to Adaptive filter and obtained denoised wave form at output which are shown in Fig-2 to Fig-8,and We compared SNR at input and Output for different window combinations which are shown from Table-1.From the above discussions it is concluded that Binary LMS Algorithm was given better Response in terms of SNR and Enhancement of Noise signal from noised input signal.and Binary LMS also provides fast convergence and also mean square error will also be smaller compare to LMS.

\section{REFERENCES}

[1] Saurabh Singh Rajput, Dr.S.S. Bhadauria-IMPLEMENTATION OF FIR FILTER USING EFFICIENT WINDOW FUNCTION AND ITSAPPLICATION IN FILTERING A SPEECH SIGNAL-International Journal of Electrical,Electronics and mechanical control.

[2] Sonika Gupta, Aman Panghal- Performance Analysis of FIR Filter Design by Using Rectangular, Hanning and Hamming Windows Methods- International Journal of Advanced Research in Computer Science and Software Engineering- Volume 2, Issue 6, June 2012ISSN: $2277128 \mathrm{X}$

[3]. TAO ZHANG- RESEARCH ON DESIGN FIR DIGITAL FILTER USING MATLAB AND WINDOW FUNCTION METHODJournal of Theoretical and Applied Information Technology- 10th February 2013. Vol. 48 No.1

[4]. R.W.Schafer,L.R.Rabiner,O.Herrmann-"FIR digital filter banks for speech analysis"-The Bell system Technical Journalvol:54,no:3, march 1979.

[5]. Mridula Malhotra, The Performance Evaluation of Window Functions and Application to FIR Filter Design-International Journal of Scientific \& Engineering Research, Volume 2, Issue 12, December-2011 1 ISSN 2229-5518

[6] J. Parak, J. Havlik-ECG SIGNAL PROCESSING AND HEART RATEFREQUENCY DETECTION METHODS

[7] P Bonizzi, O Meste, V Zarzoso-Spectral analysis of atrial signals directly from surface ECG exploiting compressed spectrum.

[8] LEIF SO * RNMO, PABLO LAGUNA-ELECTROCARDIOGRAM (ECG) SIGNALPROCESSING

[9] R. Sassi, L.T. Mainardi, P. Maison-Blanche3 and S. CeruttiESTIMATION OF SPECTRAL PARAMETERS OF RESIDUAL ECG SIGNAL DURING ATRIAL FIBRILLATION USING AUTOREGRESSIVE MODELS.

[10] CN Nowak, G Fischer, L Wieser, B Tilg, HU StrohmengerFrequency Spectrum of the Intracardiac and Body SurfaceECG during Ventricular Fibrillation - a Computer Model Study

[11] Jong-Yih Lin _, Ching-Wen Liao "New IIR filter-based adaptive algorithm in active noise control applications:

Commutation error-introduced LMS algorithm and associated convergenceassessment by a deterministic approachI"- Automatica 44 (2008) 2916 2922-Elsevier Publications 
INTERNATIONAL JOURNAL OF INNOVATIVE RESEARCH IN ELECTRICAL, ELECTRONICS, INSTRUMENTATION AND CONTROL ENGINEERING Vol. 2, Issue 12, December 2014

[12] Ibtissam Constantin,Regis Lengelle-"performance Analysis of Kernal Adaptive Filters Based on LMS Algorithm"-procedia Computer science 20(2013) 39-45, sciencdirect.

[13] Michael A. Vaudrey, William T. Baumann, William R. Saunders"Stability and operatingconstraints of adaptive LMS-basedfeedback control"-Automatics-Sciencdirect

[14] Wang An-dong, Liu Lan ,Wei Qin-“An Adaptive Morphologic Filter Applied to ECG De-noising and Extraction of R Peak at Realtime" 2012 AASRI Conference on Computational Intelligence and Bioinformatics,Elsevier

[15] Kaibo Hu*, Yaxuan Liu-“Adaptive Noise Cancellation Method for Fiber Optic Gyroscope"- 2012 International Workshop on Information and Electronics Engineering (IWIEE),Elsevier

[16] .V.Muralidhar, V. L. N.sastry D, S.K.Nayak, "Interpretation of Dirichlet,Bartlett, Hanning and Hamming windows using Fractional FourierTransform", International Journal of Scientific \& Engineering Research,vol. 4, issue 6, June-2013.

[17] Muralidhar, P. V., D. V. L. N. Sastry, and S. K. Nayak. "Spectral Analysis of Shadow Window-FIR Filters." Int. Conf. on Advances in Communication, Network, and Computing. 2013.]

[18] P.V.Muralidhar,A.S.Rao,S.K.Nayak,"Spectral Interpretation of Sinusoidal wave using Fractional Fourier Transform based FIR Window functions"-International Review of computers and software 4.6(2009).

[19] Muralidhar.P.V.,A.S.Srinivasa Rao,Dr.S.K.Nayak" Fractional Fourier Transform based FIR window functions"-Proceedings of II nd Interrnational Conference RSPS-2010 sponsored by IEEE(Hyderabad section).

[20] Muralidhar, P. V., D. Nataraj, V. Iokesh Raju, and S. K. Naik. "Implementation of different FIR high pass filters using fractional Kaiser window." In Signal Processing Systems (ICSPS), 2010 2nd International Conference on, vol. 2, pp. V2-651. IEEE, 2010. 